\title{
Dos nuevas subespecies de Linaria amethystea (Vent.) Hoffmanns. \& Link (Plantaginaceae) en el sur de la Península Ibérica
}

\author{
Federico Casimiro-Soriguer Solanas \& Baltasar Cabezudo
}

Área de Botánica, Universidad de Málaga.

\author{
Correspondencia \\ F. Casimiro-Soriguer \\ e-mail: fedeque@hotmail.com \\ Recibido: 22 julio 2019 \\ Aceptado: 11 septiembre 2019 \\ Publicado on-line: 19 septiembre 2019 \\ Editado por: A.V. Pérez-Latorre
}

\begin{abstract}
Resumen
Se propone una nueva combinación nomenclatural Linaria amethystea subsp. albiflora (Boiss.) Casimiro-Soriguer Solanas \& Cabezudo y un nuevo taxón, L. amethystea subsp. toloxense Casimiro-Soriguer Solanas \& Cabezudo. Se aclaran las relaciones de ambos taxones con el resto de subespecies de $L$. amethystea presentes en el sur de la Península lbérica y se aporta una clave de identificación para todas las subespecies reconocidas en la Península Ibérica dentro del complejo de L. amethystea.
\end{abstract}

Palabras clave: Linaria, taxonomía, Andalucía, Sierra de las Nieves

\begin{abstract}
Two new subspecies of Linaria amethystea (Vent.) Hoffmanns. \& Link (Plantaginaceae) from the southern Iberian Peninsula

A new nomenclatural combination Linaria amethystea subsp. albiflora and a new taxa $L$. amethystea subsp. toloxense are proposed. The relationships of both taxa with the rest of subspecies of $L$. amethystea present in the south of the Iberian Peninsula are clarified an identification key for all the subspecies recognized in the Iberian Peninsula within the complex of $L$. amethystea is provided.
\end{abstract}

Key words: Linaria, taxonomy, Andalusia, Sierra de las Nieves

\section{Introducción}

Linaria amethystea (Vent.) Hoffman. \& Link, es una especie muy variable (Valdés, 1970; Sáez \& Bernal, 2009), variación que afecta tanto a caracteres vegetativos (tamaño de la planta, morfología de las hojas, etc.) como reproductivos (morfología y coloración de las flores, tipo y densidad del indumento en la inflorescencia, etc.). Tiene una distribución ibero-norteafricana y se han reconocido 4 subespecies (Valdés 2002; Sáez \& Bernal, 2009): L. amethystea subsp. amethystea, endémica de la Península Ibérica y que se distribuye por toda el área peninsular de la especie, L. amethystea subsp. multipunctata (Brotero) Chater \& D.A. Webb, endemismo del extremo occidental de la Península Ibérica (Beira Litoral y Estremadura portuguesa), L. amethystea subsp. ignescens (Kunze) D. A. Sutton, endémica del suroeste peninsular (provincia de Cádiz, España) y $L$. amethystea subsp. broussonetii
(Poir.) Malato-Beliz, endémica del noroeste de África (norte de Marruecos). Las subspecies multipunctata e ignescens tienen una distribución simpátrica con la subsp. amethystea mientras que la subsp. broussonetii es el único taxón de la especie localizado fuera de la Península Ibérica.

En un estudio que estamos realizando sobre la diversidad vegetal de la Serranía de Ronda (Málaga, España), la totalidad del material recolectado, incluible dentro de $L$. amethystea s.I., no encajaba claramente con las descripciones de los taxones incluidos en esta especie y recogidos en las obras de referencia Flora iberica (Castroviejo, 1986-2019) y Flora Vascular de Andalucía Oriental (Blanca et al., 2011), razón por la cual se decidió hacer un estudio de estas poblaciones. Para ello se ha analizado el material recolectado en la zona de estudio y revisado material de herbario de esta especie recolectado fundamentalmente en el sur de la Península Ibérica. 


\section{Material y método}

Hemos estudiado material de L. amethystea (s.l.) depositado en los siguientes herbarios: COA, COAF, G, GDA, MA, MGC y SEV (acrónimos según Thiers 2018), en total 153 pliegos (Anexo1), y se han revisado síntesis de referencia del género Linaria para la Península Ibérica (Sáez \& Bernal, 2009; Valdés, 1970) y para el sur de la Península Ibérica (Valdés, 1987; Sáez \& Sainz, 2011). Todo el material recolectado en la zona de estudio se ha depositado en el herbario MGC de la Universidad de Málaga (España).

\section{Resultados y discusión}

En todos los trabajos consultados se describe a L. amethystea (s.l.) como una planta glabra excepto en los cálices (Hoffmannsegg \& Link 1:253 (1811); Cavanilles 1:20 (1791); Lamarck 4: 353 (1797); Sutton, 1988; Devesa, 1995; Willkomm in Willkomm \& Lange 3: 566-567 (1878); Coutinho, 1939; Merino 2: 70 (1906); Sáez \& Bernal, 2009; Chater et al., 1972), o incluso totalmente glabra (Sáez \& Bernal, 2009). Únicamente Valdés (1970, 1987) describe a $L$. amethystea como una planta que puede ser pubescente glandulosa.

Sin embargo, como resultado del estudio realizado, se observa una modificación en la presencia de indumento y en la coloración de la corola en relación con la distribución geográfica. Desde la depresión del río Guadalquivir hacia el sur, las poblaciones empiezan a presentar pubescencia tanto en tallos como en hojas, así como una coloración predominantemente blanca en la corola, caracteres constantes en las poblaciones de la especie que habitan en la Cordillera Bética. Al norte de la depresión del Guadalquivir las poblaciones son glabras en tallos y hojas y tienen corolas predominantemente azul-violeta, raramente blancas. Este marcado patrón geográfico nos ha parecido lo suficientemente significativo como para proponer como subespecies independientes a estas poblaciones al sur del Guadalquivir caracterizadas por ser pubescente-glandulosas en tallos y hojas y tener corolas blancas. También la morfología del espolón parece seguir el mismo patrón geográfico, las poblaciones al norte de la depresión del Guadalquivir tienen los espolones rectos o curvados en su extremo hacia atrás, sin embargo, en las poblaciones al sur del Guadalquivir presentan espolones rectos o curvados hacia adelante, raramente hacia atrás. En la transición entre los pisos supra y oromediterráneo de la Sierra de las Nieves (Málaga) hemos localizado unas poblaciones similares a las generales del sur del Guadalquivir, pero con claras diferencias en la morfología de la inflorescencia y en la densidad de la pubescencia. En base a lo anterior se propone una nueva combinación y se describe un nuevo taxón para las poblaciones béticas.

Linaria amethystea (Vent.) Hoffmanns. \& Link subsp. albiflora (Boiss.) Casimiro-Soriguer Solanas \& Cabezudo stat. nov.

$=L$. amethystea var. albiflora Boiss., Voy. Bot. Espagne 2: 464 (1841).

Typus: In arenis maritimis, Gibraltariae (Lectotypus, G 00356857*, - Designado por Valdés (1970: 239) -); Isolectotypus, G 00356858*, 00356859*, 00356861*, 00356862* ${ }^{*}$ 356863*, MA110162) *Catalogue des herbiers de Genève $(\mathrm{CHG})$. Conservatoire \& Jardin botaniques de la Ville de Genève, http://www.ville-ge.ch/musinfo/bd/ cjb/chg (Último acceso junio 2019).

Diagnosis: Plantas anuales, pelosoglandulosas en tallos y hojas con inflorescencias peloso-glandulosas, densas en la floración y netamente alargadas en la fructificación, corolas blancas con espolón de longitud mayor o igual al resto de la corola, el espolón de color violáceo, semillas con disco tuberculado y ala estrecha y engrosada, concoloras cuando están totalmente maduras, de gris oscuro a negro (Fig. 3).

Distribución y hábitat: Se distribuye en el sur de la Península Ibérica, abarcando los territorios al sur del río Guadalquivir, ampliamente repartida por la Cordillera Bética y áreas limítrofes, en las provincias de Cádiz, Córdoba, Granada, Jaén y Málaga.

Pastizales de terófitos en hábitats variados, desde zonas cultivadas a frecuentemente en claros de matorral, parece tener preferencia por sustratos carbonatados, de 0-1000 (1300) m.

Observaciones: Boissier (1841), describió la var. albiflora del Peñón de Gibraltar y de la Serranía de Ronda, ampliando su distribución a los alrededores de Madrid, citando a Cavanilles (1791). Hemos consultado el material tipo de la var. albiflora (G, MA) y todo el material es pubescenteglanduloso en hojas y tallos. Por tanto, la mayoría de las poblaciones béticas entran dentro del criterio con el que Boissier eligió el tipo de la variedad. En la descripción de la variedad, Boissier considera que las diferencias más significativas de este taxon son la corola blanca y los espolones relativamente cortos, ignorando la presencia de abundante indumento pubescente-glanduloso, razón por la 
que sinonimiza equivocadamente su variedad al Anthirrinum bipunctatum Cav. (Cavanilles, 1791: 20), ya que, en su descripción, Cavanilles indica claramente que las plantas que recoge bajo ese nombre tienen flores blancas, espolones de longitud similar al resto de la corola y hojas glabras. Hemos revisado material recolectado en Aranjuez e identificado como var. albiflora y efectivamente presenta hojas y tallos glabros.

Linaria amethystea (Vent.) Hoffmanns. \& Link subsp. toloxense Casimiro-Soriguer Solanas \& Cabezudo, subsp. nov. (Figs. 1A, 1C).

Typus. España: Málaga, Tolox, Parque
Natural Sierra de las Nieves, desde el Puerto de los Pilones al Cerro de la Cueva del Oso, 1700$1750 \mathrm{~m}$. Comunidad de sabinares rastre-ros, quejigales, piornales espinosos y espinares. Calizas. 26/06/2018. B. Cabezudo, F. Soriguer \& A.V. Pérez Latorre. (MGC 89000, Holotypus; MGC 88999, 89001, Isotypus).

Diagnosis: Diferente del resto de taxones incluidos en $L$. amethystea por ser densamente glandular-pubescente en tallos, hojas e inflorescencias, presentar inflorescencias congestas en flor y en fruto, hasta de $1 \mathrm{~cm}$ de longitud, y tener flores blancas con espolones cortos (Fig. 1).
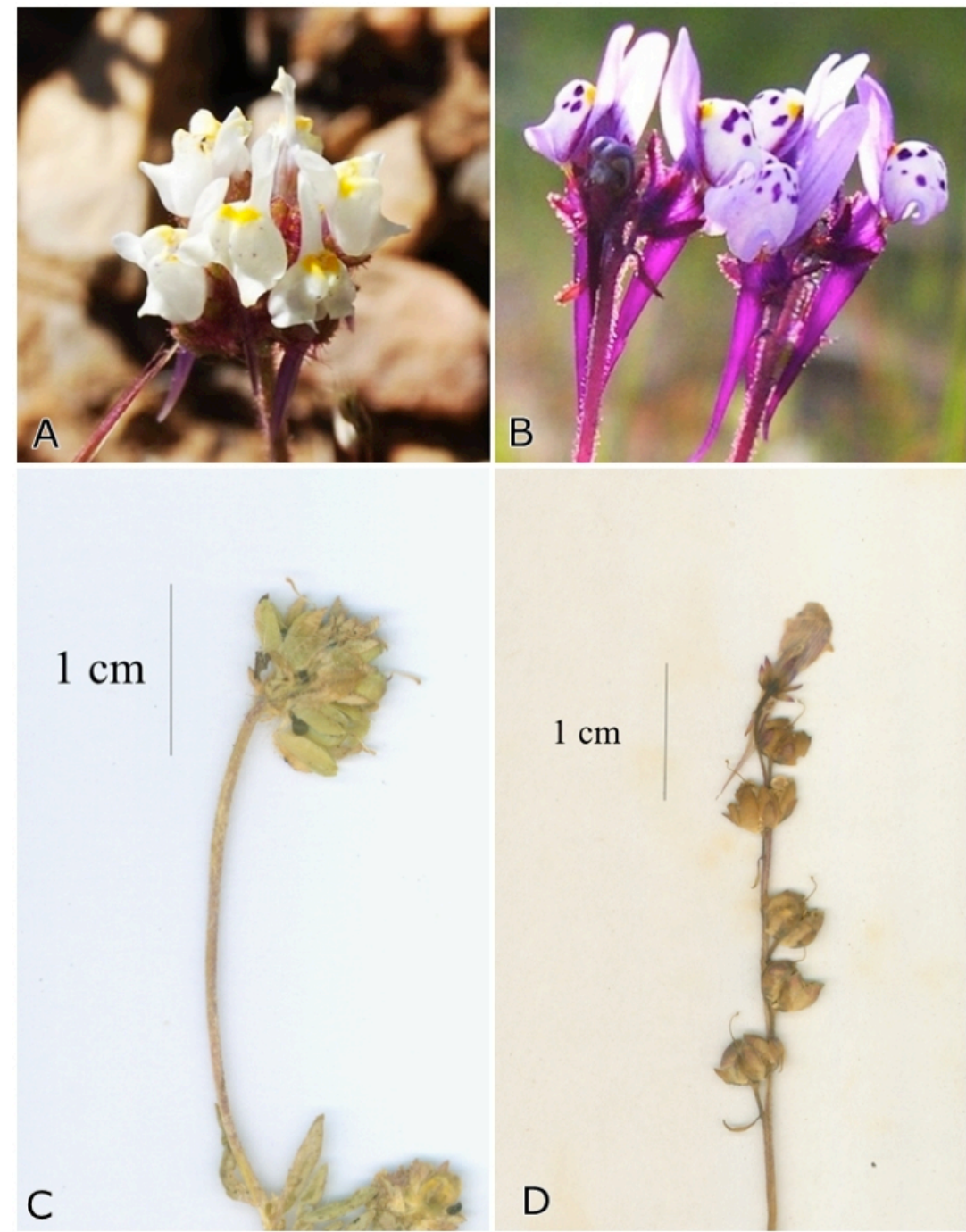

Figura 1. Diferencias en la inflorescencia en flor y en fruto entre L. amethystea subsp. toloxense (A. Detalle de la inflorescencia en flor, Quejigal de Tolox, P.N. Sierra de las Nieves (Málaga); C. Detalle de la inflorescencia en fruto, holotypus MGC 89000) y L. amethystea subsp. amethystea (B. Detalle de la inflorescencia en flor, Sierra de Andújar, Sierra Morena (Jaén) -Imagen cedida por G. Blanca-; D. Detalle de la inflorescencia en fruto, Sierra de Aracena (Huelva) MGC 10271).

Figure 1. Differences between inflorescences in flower and fruit of L. amethystea subsp. toloxense (A. Inflorescence in flower; $C$. Inflorescence in fruit) and L. amethystea subsp. amethystea (B. Inflorescence in flower; $D$. Inflorescence in fruit). 


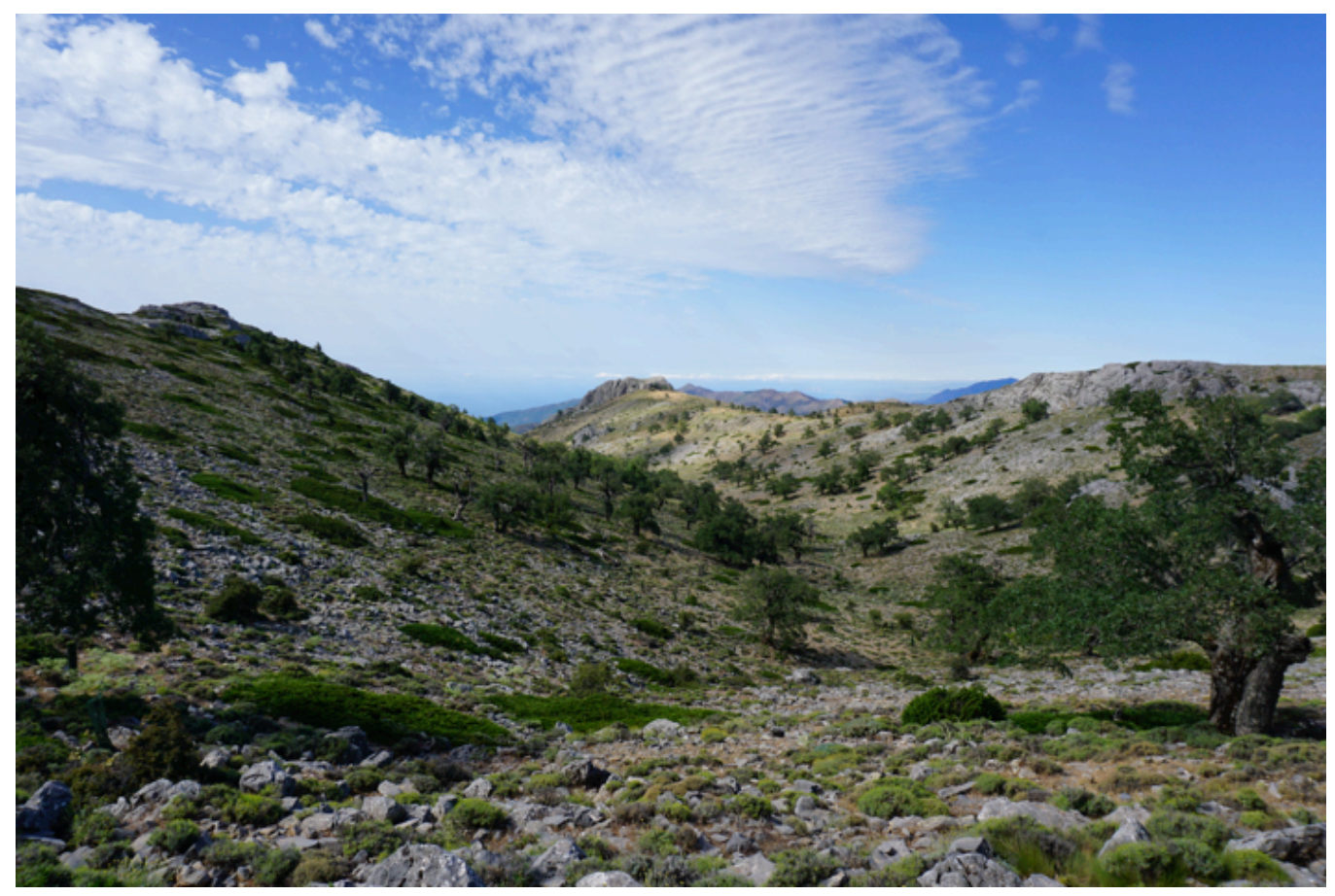

Figura 2. Hábitat de L. amethystea subsp. toloxense, Quejigal de Tolox, P.N. Sierra de las Nieves (Málaga, España).

Figure 2. Habitat of L. amethystea subsp. toloxense.

Diagnosis: Differs from the other taxa within L. amethystea by having stems, leaves and inflorescences densely glandular-pubescent, inflorescences dense in flower and fruit up to $1 \mathrm{~cm}$ long, and white flowers with short spur (Fig. 1).

Descripción: Planta anual, pubescenteglandulosa. Tallos de hasta $8(10) \mathrm{cm}$, pubescente-glandulos, pelos 0,1-0,2 $\mathrm{mm}$, glandulíferos. Hojas de los tallos fértiles 2-8 $x$ $0,5-2 \mathrm{~mm}$, linear-lanceoladas, planas, alternas, con abundante indumento igual al de los tallos. Inflorescencias $(0,5)$ 0,6-0,8 $(1,1) \mathrm{cm}$, con 1-5 flores, densas en la antesis y en la fructificación. Brácteas 3-4 x 0,5 mm, lineares, densamente peloso-glandulosas, netamente más largas que los pedicelos. Pedicelos 1-1,5 (2,2) mm, peloso-glandulosos. Cáliz densamente pelosoglanduloso con sépalos 2,5- 4 × 0,6-0,8 mm, subiguales, aunque el superior puede ser algo más largo. Indumento de cálices, pedicelos y brácteas formado por pelos de (05) 0,8-1,2 $(1,5)$ $\mathrm{mm}$, pluricelulares, hialinos, que presentan, la mayoría, células rojas intercaladas que le dan un aspecto rojizo a la inflorescencia, el indumento es muy denso en la base del cáliz dándole un aspecto viscoso. Corola $(10,5) 13-15$ (18) mm, blanca con escasas manchas violetas en el labio inferior, glabra excepto en la garganta, paladar amarillo en su base. Espolón 6-9 mm, de poco más largo a igual que el resto de la corola, recto o curvado hacia adelante, normalmente violáceo, a veces blanco. Cápsula $4 \mathrm{~mm}$ de diámetro, globosas con indumento por toda su superficie de pelos cortos glandulíferos. Semillas 1-1,3 $\mathrm{mm}$ de diámetro, aladas, orbiculares, plano convexas con ala de $0,25 \mathrm{~mm}$, engrosada. Disco esparcidamente tuberculado, disco y ala del mismo color, gris oscuro cuando están totalmente maduras.

Etimología: El epíteto toloxense proviene de Toloxeño, gentilicio de los naturales de Tolox (Málaga).

Distribucion y hábitat: Endémica de la Sierra de Tolox, incluida en el P. N, Sierra de las Nieves (Málaga) en la Serranía de Ronda (Andalucía, España). Se encuentra exclusivamente entre los pisos supra y oromediterraneo, de 1650 a 1800 m.s.n.m., ocupando un territorio de aproximadamente $7,5 \mathrm{Km}^{2}$. Forma parte de pastizales de terófitos asociados a suelos provenientes de la descalcificación de calizas, en el seno de formaciones abiertas de Quercus faginea subsp. alpestris (Boiss.) Maire, Juniperus sabina L. y J. communis subsp. hemisphaerica (K. Presl) Nyman (Fig. 2). 


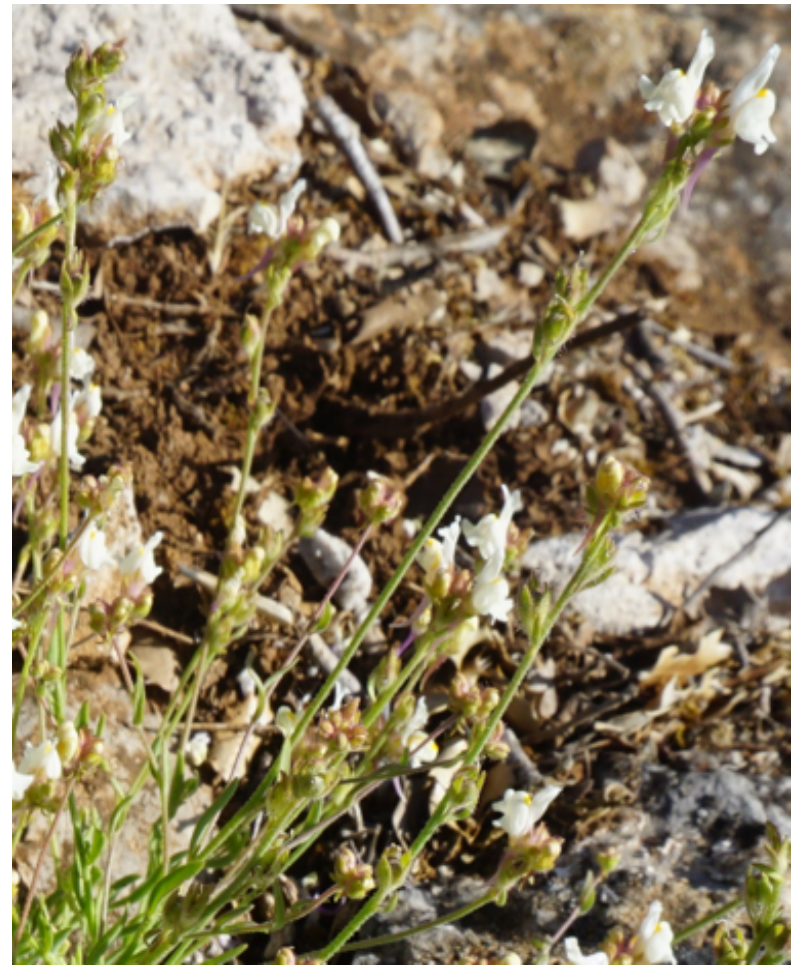

Figura 3. Inflorescencias netamente alargadas comenzando a fructificar de L. amethystea subsp. albiflora (P.N. Sierra de las Nieves, Conejeras, 1000 m.s.m.).

Figure 3. Clearly elongated Inflorescences in fruit of $L$. amethystea subsp. albiflora.

Fenología: Terófito de vida corta. Florece de Mayo a Junio y fructifica y dispersa en Junio.

Observaciones: L. amethystea subsp. toloxense está muy relaccionada con L. amethystea subsp. albiflora, pero mantiene las inflorescencias congestas en la fructificación, carácter que solo comparte con la subsp. multipunctata (cf. Sáez \& Bernal, 2009). La subsp. toloxense presenta de forma constante una serie de caracteres diferenciales como flores pequeñas, blancas y con espolones de longitud similar a la corola, es densamente glandular pubescente en todos sus órganos, excepto las corolas, y sus inflorescencias e infrutescencias son cortas y de igual tamaño. La población que integra a la nueva subespecie propuesta es la situada a mayor altitud en el sur de la Península Ibérica (Valdés, 1987; Sáez \& Sainz, 2011), estando aislada del resto de poblaciones al ocupar la zona supra-oromediterránea de la serranía de Ronda (cf. Pérez Latorre et al., 1998), mientras que el resto de poblaciones de L. amethystea subsp. albiflora de esta zona se distribuyen en el piso termo y mesomediterráneo.
Clave de identificación de las subespecies de $L$. amethystea de la Península Ibérica (basada en Sáez \& Bernal, 2009 y reestructurada para incluir los nuevos taxones):

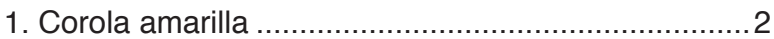

1. Corola azul-violeta o blanca .................................. 3

2. Corola 18-28 $\mathrm{mm}$, por lo general con manchas violetas; inflorescencia \pm densa en la antesis y en la fructificación; semillas 1,2-2 × 1-1,8 mm. subsp. multipunctata

2. Corola $15-19(23) \mathrm{mm}$, sin manchas violetas; inflorescencia \pm laxa en la antesis y en la fructificación; semillas 0,9-1,3 × 0,9-1,1 mm......... subsp. ignescens

3. Plantas glabras en tallos y hojas. Corola azul violeta o blanca..............................................subsp. amethystea

3. Plantas glandular-pubescente en tallos y hojas. Corola blanca.....

4

4. Inflorescencia densa en la antesis, y netamente alargada en la fructificación de hasta $14 \mathrm{~cm}$

..subsp. albiflora

4. Inflorescencia densa en la antesis y en la fructificación de hasta $0,8-(1,1) \mathrm{cm}$..................... subsp. toloxense

\section{Agradecimientos}

Este trabajo se ha realizado en el marco de un convenio entre la Junta de Andalucía y la Universidad de Málaga (UMA), bajo el título de "Estudio de la Flora y Vegetación del futuro Parque Nacional Sierra de las Nieves". Agradecemos las facilidades dadas para el trabajo de campo por el director del Parque Natural Sierra de las Nieves y su equipo de agentes de medio ambiente, así como a todos los miembros del grupo de investigación RNM-115 (UMA) de la Junta de Andalucía que participan en este convenio. Agradecemos también al personal de los herbarios COA, COAF, GDA, MA, MGC y SEV por el envío del material. A los revisores anónimos de este trabajo por sus correcciones y aportaciones que han ayudado a mejorarlo. A G. Blanca por cedernos la imagen de la inflorescencia de L. amethystea subsp. amethystea.

\section{Bibliografía}

Blanca, G., Cabezudo, B., Cueto, M., Salazar, C. \& Morales Torres, C. (Eds). (2011). Flora Vascular de Andalucía Oriental, $2^{\mathrm{a}}$ ed. Universidades de Almería, Granada, Jaén y Málaga, Granada, 1751 pp.

Boissier, P.E. (1841). Voyage botanique dans le midi de l'Espagne pendant l'année 1837, vol. 2. Paris, 757 pp.

Cavanilles, A.J. (1791). Icones et descriptiones plantarum, quae aut sponte in Hispania crescunt, aut in hortis hospitantur, vol. 1. Lazaro Gayguer, Madrid. $172 \mathrm{pp}$.

Chater O., Valdés B., \& Webb D.A. (1972). Linaria Mill. In 
Tutin T.G., Heywood V.H., Burges N.A., Moore D.M., Valentine, D.H., Walters, S.M. \& Webb, D.A. (Eds.) Flora Europaea, vol. 3. Cambridge University Press, Cambridge, pp.226-236.

Castroviejo, S. (Ed.) (1986-2019). Flora iberica, vols. 1-21. Real Jardín Botánico, CSIC, Madrid.

Coutinho, A. (1939). Flora de Portugal (2. ${ }^{a}$ edição) Bertrand Ltd., Lisboa, 938 pp.

Devesa, J.A. (1995). Vegetación y flora de Extremadura. Universitas ed. Badajoz, 773 pp.

Hoffmannsegg, J.C. \& Link, J.H.F. (1811). Flore Portugaise, vol.1. Amelang, Berlín, 536 pp.

Lamarck J.B. (1797). Encyclopédie Méthodique Botanique, vol. 4. Agasse, París,764 pp.

Merino, B. (1905-1909). Flora descriptiva é ilustrada de Galicia, vol.2. Galaica Santiago de Compostela, 635 pp.

Pérez Latorre, A. V., P. Navas, D. Navas, Y. Gil \& Cabezudo B. (1998). Datos sobre flora y vegetación de la Serranía de Ronda (Málaga, España). Acta Botanica Malacitana 23, 149-191.

Sáez, LI. \& Bernal, M. (2009). Linaria Mill. In Castroviejo S. (Coord.) Flora Iberica vol.13 (pp.232-324). Real Jardín Botánico. CSIC, Madrid.

Sáez, LI. \& Sainz, M. (2011). Linaria Mill. In Blanca, G., Cabezudo, B., Cueto M., Morales Torres, C. \& Salazar, C. (Eds.). Flora vascular de Andalucía Oriental, $2^{a}$ edición (pp. 1233-1245). Universidades de Granada, Almería, Jaén y Málaga, Granada.

Sutton, D.A. (1988). A Revision of the Tribe Antirrhineae (Scrophulariaceae). British Museum (Natural History), London, $550 \mathrm{pp}$.

Thiers, B. (2019). Index Herbariorum: A global directory of public herbaria and associated staff. New York Botanical Garden's virtual Herbarium. available from: http://sweetgum.nybg.org/ science/ih/ (último acceso mayo de 2019). Valdés, B. (1970). Revisión de las especies europeas de Linaria con semillas aladas. Publicaciones de la Universidad de Sevilla, Ser. Ciencias- $n^{\circ} 7$, Sevilla, 288 pp.

Valdés, B. (1987). Linaria Mill. In Valdés, B., Talavera, S. \& Galiano, E.F. (Eds.) Flora Vascular de Andalucía Occidental, vol.2 (pp.508-522) Ketres, Barcelona.

Valdés, B. (2002) Linaria Mill. In B. Vadés, M.Rejdali, A. Achhal El Kadmiri, J.L. Jury, J.M.Montserrat (eds.) Catalogue des plantes vasculaires du Nord du Maroc, vol. 1 (pp. 569-575). CSIC. Madrid, pp. 569-575.

Willkomm, M. \& Lange, J. (1878). Prodromus Florae Hispanicae, vol. 3. E. Schweizerbart, Stuttgart, 680 pp.

\section{Anexo1}

\section{Linaria amethystea subsp. toloxense (paratypus)}

ESPAÑA. Málaga: Tolox, Parque Natural Sierra de las Nieves, vereda al Torrecilla, $1720 \mathrm{~m}$. Pastizal anual, calizas, 12/06/1997, A.V. Pérez Latorre, P. Navas, Y. Gil \& D. Navas (MGC 46205); ibídem, base de la Torrecilla, 1700 m, 23/06/1988, B. Díez Garretas \& A. Asensi (MGC 24701); ibídem, entre el Puerto de los Pilones y el pozo de nieve, $1730 \mathrm{~m}$, pastizales anuales, calizas y arcillas, 22/05/2015, B. Cabezudo \& F. Soriguer (MGC 81078); ibídem, vereda del Puerto de los Pilones al Peñón de Enamorados, 1700-1750 m, quejigal adehesado, pastizales y piornales espinosos, calizas y arcillas, B. Cabezudo, F. Soriguer \& A.V. Pérez Latorre (MGC 85782); ibídem, desde el Puerto de los Pilones al Pilar de Tolox, 1700m, 13/06/2018, F. Soriguer \& N. Hidalgo (MGC 88727).

\section{Linaria amethystea subsp. albiflora}

ESPAÑA. Cádiz: Grazalema, Sierra del Endrinal, 100-1200 m, 12/07/1984, A. Aparicio \& S. Silvestre (SEV 160732-2); ibídem, Sierra del Caillo, 22/05/1974, S. Silvestre (SEV 100232); ibídem, Sierra de Grazalema, 09/06/1993, A. Aparicio, M.A. Carrasco \& M. Velayos (SEV 238743); ibídem, Sierra del Endrinal, 12/07/1984, A. Aparicio \& S. Silvestre (SEV 160732); ibídem, Puerto de las Palomas, 19/07/1990 (SEV 160593); ibídem, Sierra de Zafalgar, 21704/1989, P.E.Gibbs, S. Talavera et al. (SEV 136660); ibídem, caserio de Campobuche, 13/06/1980, M.J. Gallego et al. (SEV244643); ibídem, Sierra del Pinar, 15/07/198, S. Silvestre (SEV 244641); ibídem, de Grazalema a Ronda, 19/03/1969, P.E.Gibbs (SEV 4063); Arcos de la Frontera, El Mosle, 15/05/1975, S. Silvestre \& S. Talavera (SEV 101940); ibídem, 27/04/2007, J.L. Díaz Aragón \& O. Comino Matas (MGC 66608); Bornos, 01/04/1993, M. Arista (SEV 135294); ibídem, 15/03/1973, J.C. Escudero (SEV 101662); ibídem, 22/04/1976, Brinton-Lee (SEV 90058); Benaocaz, Veradillo, 15/06/1971, Brinton-Lee (SEV 81205); Gibraltar, Catalan Bay, 16/05/1985, J. Bensusan et al. (SEV 124911); San Roque, Sierra Carbonera, 125150 m, 31/01/2003, J.A. García Rojas (MGC 55423); Gibraltar, in arenosis regionis calidae, 06/1837. Boissier (MA 110162*). Cordoba: Puente Genil, Sotogordo, río Genil, 29/04/1987, F. García Montoya (COFC 59303); ibídem, Cerro Herriza, 12/03/1987, F. García Montoya, (COFC 59304); ibídem, laguna de Tiscar, 30/04/1986, F. García Montoya (COFC 59473); ibídem, 11/03/1986 (COFC 58896); ibídem, Sierra del Castillo, castillo Anzur, 02/05/1986, F. García Montoya \& J.M. Muñoz (COFC 58953); Córdoba, casco urbano, 14/02/1991, P. Cariñanos (COFC 56942); ibídem, 14/02/1991, C. Galán \& Ruíz de Clavijo (COFC 52460); Montemayor, 09/05/1983, J.M. Montoro (COA 07266); Aguilar de la Fontera, Fuente Laguna, 10/03/1982, A. Pujadas et al. (COA 07268); De Bujalance a Cañete, 23/03/1983, A. Pujadas (COA 07269); De Lucena a Puente Genil, 07/02/1985, F. García Montoya (COFC 58687). Granada: Pinos Puente, Sierra Elvira, 12/02/1980, M. Ladero \& J. Hurtado (GDA 10790); ibídem, cantera, 23/04/1942, J. Muñoz Medina (GDA 39348); ibídem, 03/03/1988 (GDA 42486); Granada, Generalife, 12/03/1941, J.M.Muñoz (GDA 39344); ibídem, frente al cementerio, 09/04/1988, (GDA 32800); ibídem, Silla del Moro, 03/1971, C. Morales (GDA 2190); ibídem, Parque de Invierno, 04/1963, J. Varo (GDA 3133); ibídem, alrededores de Granada, 02/1966, J. Varo (GDA 2193); Padul, Cerro de Los Molinos, 750 m, 09/03/1989, López Nieto, (GDA 22924); ibídem, 05/1987, J. Martín (GDA 26934); Ogijares, 12/03/1988, Angeles 
Ibañez (GDA 32839); Armilla, base aérea, 06/05/1989, M.T.Vizoso \& J.C. de la Rosa (GDA 32462); Loja, campos de Loja, 05/1974, J. Varo \& J.A. Gil (GDA 2189); ibídem, Sierra de Loja, El Cofín, 600 m,05/04/1989, M.V. García et al. (GDA 33648); ibídem, proximidades de Loja, 02/03/1974, A. Asensi \& B. Díez (MGC 1388); ibídem, $2 \mathrm{~km} \mathrm{Al} \mathrm{oeste,} \mathrm{07/04/1979,} \mathrm{Stellan} \mathrm{Holmdahl} \mathrm{(MGC}$ 49840); Cullar Vega, Vegas del Genil, 10/03/1996, L. Roca Castillo (GDA 40338); Llanos de Armilla, 04/1973 (MA 752139); Aeropuerto de armilla, C. Morales et al. (MA 422500); La Peza, 14/05/1971, Fernández Casas (MA 422252); ibídem (MA 422252); subida al Llano de la perdiz, 700m, 20/02/1978, Molero Mesa (MA 429687); Alhendín, Montevives, 840 m, 28704/1979, F. Pérez Raya (MA 426520); Alhama de Granada, Sierra Tejeda, Los Rodaderos, 1200m, 05/06/2007, A.V. Pérez Latorre et al. (MGC 66521); El Tacón, 07/05/1983, A. Asensi \& B. Díez Garretas (MGC 1246). Jaén: Torredelcampo, 01/04/1974, G. Blanca (GDA 2191); Úbeda, Sierra de las Cabras, 500 m, 10/05/1982, C. Fernández \& J. Cobos (MA 426619). Málaga: Sierra de las Nieves, 900m, 24/06/1966, F. Gettlife et al. (SEV 3818); ibídem, Cortijo de San Luis, 900 m, 24705/1966, F. Getliffe at al. (SEV 3823); Entre Ronda y Cartajima, Peña Rodá, 850-1150 m, 18/06/1974, S. Talavera \& B. Valdés (SEV 95729); Arriate,25/03/1979, J.Díez \& T. Luque (SEV 94711); Cortes de la Frontera, Sierra de los Pinos, 700-1000 m, 29/05/1984, A. Aparicio \& S. Silvestre (SEV 1607302); ibídem, (SEV 160730); ibídem, 03/05/1990 (SEV 16059); Parauta, Cerro Serranía, 1100m, -sin fecha-, $D$. Montilla (SEV 162475); Ronda, Prope Arenal de Ronda, 16/04/1922, E. Gros (SEV 112862); ibídem, Ermita de la Cabeza, 640 m, D. Montilla (SEV 162474); ibídem, próximo a Casa Rico, 750 m, 12/02/1989, D. Montilla (MGC 40545); Genalguacil, Sierra Bermeja,1300m, 14/06/2000, B. Díez garretas \& A. Asensi (MGC 47952); Ibídem, Pico de los Reales, 1400m, 11/06/1996, $P$. Navas et al. (MGC 51406); Benaoján, Sierra del Palo, 25/05/2002, M. Becerra \& F. Sánchez Tundidor (MGC 51991); ibídem, Sierra de Benaoján, Tajos de la Moraleda, 600m, 11/03/2001, M. Becerra (MGC 51247); Antequera, Monte San Cristobal, 16/02/1980, J. Guerra (MGC 6858); Juzcar, Los Riscos, 1100m, 08/05/2004, O. Gavira \& A.V. Pérez Latorre (MGC 61265); Cartajima, Riscos de Cartajima, 1350 m, 30/05/2004, (MGC 61263); Entre los términos de Parauta y Ronda, Parque Natural Sierra de las Nieves, 920-980 m,23/05/2017, B. Cabezudo et al. (MGC 85859); Parauta, Parque Natural Sierra de las Nieves, de Conejeras al Navazo, 1050 m, 23/05/2017, B. Cabezudo et al. (MGC 85892); ibídem, cortijo del Navazo, 1030 m, 17/04/1997, Pérez Latorre et al. (MGC 46204); Coín, Sierra Alpujata, 01/03/1988, B. Cabezudo (MGC 21564).

* Imagen de alta resolución cedida por MA.

\section{Linaria amethystea subsp. amethystea}

PORTUGAL. Beira Litoral: Coimbra, Vale de Linhares, 03/03/1952, J. Matos \& A. Matos (MGC 834)

ESPAÑA. Cáceres: Carascalejo, 06/02/1966, Mayor et al. (MGC 4574). Cordoba: Guadalcazar, Finca La Era, 161m, 30/03/2006, P. Prados et al. (COA 5347);
Peñarroya, al sur, 24/04/1982, A. Pujadas (COA 07264); De Villaviciosa a Villanueva del Rey, 06/04/1986, A. Pujadas. (COA 07262); Santa Eufemia, $3 \mathrm{~km}$ al norte, 27/05/1983, A.Pujadas (COA 07261); Los Pedroches, 27/05/1983, A. Pujadas (COA 07260); Posadilla, 10/05 1983, A. Pujadas (COA 07259); Villanueva de Cordoba, a $20 \mathrm{~km}$ de Obejo, 26/04/1983, A. Pujadas (COA 07265); ibídem, arroyo de La Reina, 18/04/1980, J.A. Varela (COFC 48038); Cordoba, La Palomera, 17/03/2011, $M$. Lopez et al. (COA 55152); Fuente Obejuna, carretera de Cazalla, 16/05/1981, L. Corral \& P. Fernández (COFC 44253); Hornachuelos, mina de la plata, 18/03/1981, P. Fernández \& I. Porras (COFC 44254); El Cabril, cementerio nuclear, río Bembézar, 24/04/1981, $E$. Dominguez et al. (COFC 44255); Belalcázar, barrancos, 09/05/2006, J. González (COFC 29853); ibídem, río Zujar, 14/04/1986, M. Jiménez (COFC 13868); ibídem, río Guadamatilla. 20/03/1986, M. Jiménez (COFC 13866); Los Blázquez, Pico la Navarra, 26/04/1985, $R$. Gallardo \& M. Jiménez (COFC 15924); Santa Eufemia, Peñón del Horcón, 20/03/1986, M. Jiménez (COFC 13867); Belmez, arroyo de La Parrilla, 16/05/1981, J.A. Varela (COFC 48045); Villanueva del Rey, Cerro de Doña Loba, 03/04/1979, M. Arenas et al. (COFC 49104); ibídem, arroyos del Tomillarejo y de la Reina, 01/051982, F. Infante \& J.A. Varela (COFC 59862); Espiel, arroyo de las Rodadizas, 10/03/1980, J.A. Varela (COFC 48040); Entre La Granjuela y Fuente Obejuna, cerro de las Caleras, 16/05/1981, J.A. Varela (COFC 48039); El Viso, arroyo Cabeza de la Zarza, 20/03/1976. J.A. Devesa. (COFC 3470); Arroyo de los Términos, Sierra Morena, 500 m, 03/04/1994, M. Melendo (GDA 41785); Entre Villa del Río y Cañete, 200-300 m, 05/05/1988, E. Bayón et al. (MA438259); Fuente obejuna, Valdeinfierno, 25/03/1991, Pérez Latorre et al. (MGC 31078); ibídem, Los Parchez, 25703/199, B. Cabezudo et al. (MGC 31071). Huelva: El Almendro, Camino de las Minas, 18/02/2018, Segundo Pérez Sanz (MGC 51449); ibídem, Camino de la Ermita, 03/02/2001, S. Pérez Sanz (MGC 51440); cerca de Zalamea, 380 m, 03/05/1989, B. Cabezudo et al. (MGC 25813); entre Valverde y la Palma, márgenes del Río Tinto, 04/04/1986, B. Cabezudo et al. (MGC 17962); Sierra de Aracena, entre el Repilado y Cortegana, 27/03/1979, J. Rivera \& B, Cabezudo (MGC 10271). Jaén: Chiclana de Segura, Pantano de Guadalmena, 520 m, 07/05/1988, O. Tirado (COFC 23018); ibídem (GDA 27041); ibídem (MA 554216); Parque Natural de Andujar, Tagarillar, 03/04/2003, Pérez Cacho \& A. Sánchez. (COFC 29130); Sierra Morena, Despeñaperros, 01/03/1980, Pérez Raya \& Molero Mesa (GDA 39341); El Centenillo, 26/04/1996, Dolores Luque (GDA 41233); ibídem, Garcíez, 480 m.,15/03/1987. M.A. Guirao (MA 618948); Santa Elena, entre el pueblo y Despeñaperros, 600700 m, 07/03/1988, J. Pedrol (MA 439269); Desfiladero de Despeñaperros, 800m, 16/03/1985, M. Luceño et al. (MA 376406); Andujar, de Andujar a Aldehuela, 280 m. 27703/1980, C. Fernández López (MA 715120); Villanueva de la Reina, Llanos de Selladores, $680 \mathrm{~m}$, 24/03/1993, P. Díaz (MA 651059; Aldeaquemada, cola de la Cimbarra, 650 m, 07/05/1983, C. Fernández (MA 427682). Madrid: Aranjuez,08/05/1966, Rivas-Goday \& Izco (MGC 4752). Salamanca: 04/1971, B. Díez (MGC 
833). Sevilla: Aznalcollar, salida hacía el Castillo de las Guardas, $140 \mathrm{~m}, 16 / 02 / 2001$, B.Cabezudo et al. (MGC 48802). Zamora: Castro de Alcañices, 600 m, 03/06/1981, B. Casaseca (MGC 10336).

\section{Linaria amethystea subsp. multipunctata}

PORTUGAL. Beira Litoral: Ponte de viadores, Mealhade, 08/03/1952, A. Fernandes et al. (MGC 835).

Linaria amethystea subsp. ignescens

ESPAÑA. Cádiz: Entre Jerez de la Frontera y EI Puerto de Santa María, 09/03/1978, J. Pastor et al. (SEV
117074); ibídem, (SEV 116769); ibídem, 16/04/1993, $R$. Juan \& J. Pastor (SEV 135300); ibídem, 20/03/1969, Brinton-Lee (SEV 81086); Puerto de Santa María, Cerro de San Cristobal, 10/04/1925, E. Gros (SEV 112861); ibídem, 01/02/1971, B. Cabezudo et al. (SEV 244640); ibídem, 04/03/1982, J. Devesa et al. (SEV 116770); ibídem, 24/03/1979, M.J. Díez et al. (SEV 116566); Vejer de la Frontera, 17/04/1956, V.H. Heywood (SEV 101566); Monte de En medio, 24/04/1925 -sin recolector(SEV 112860); Arcos de la Frontera, Llanos de la Huerta, 03/03/1978, F. García et al. (SEV 117507). 